INDIAN JOURNAL OF POWER \& RIVER VALLEY DEVELOPMENT www.ijprvd.info

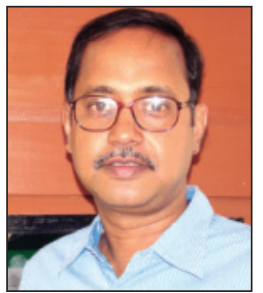

\title{
Will integrated solar-coal hybrid power plants prove to be the best foot forward for future energy in India?
}

The old technology and old coal-fired power plants today struggle under the legal and environmental burden as well societal objections. The popular demand is that both old and new electricity-generating units should have low capital costs, low fuel costs, minimal environmental impacts, and satisfy demand without concerns of intermittency. When expanding generating capacity, candidate technologies can be evaluated against criteria such as these. Alternatively, it may be possible to pair technologies in such a way that the combination addresses these criteria better than either technology individually. One such approach is to pair concentrated solar power and coal combined cycle units. Wind energy is also a candidate but we will not discuss them much here because Indian power plants seem to be more appropriate for solar integration.

Such combined units would be market-competitive if optimistic goals for capital cost and avoidable coal purchases are met. Furthermore, if used in parts with strong solar resources, these combined units could produce as much as $1 / 7$ th of national electricity generation by 2050 . Whether this adoption leads to reduced air pollutant and greenhouse gas emissions, is dependent on the technologies, it displaces.

Solar generation till the other day was cost-competitive with fossil fuels in many parts of the world (EIA 2016); the caveat is it tilted a little back in favour of coal due to the situations under pandemic. But that can again change after a temporary upset. Solar and wind technologies limit exposure to fuel price fluctuations and do not emit air pollutants during operation. However, capital costs of these technologies would be affected by the supply and demand of materials such as carbon fiber, silicon, and rare earth metals.

Old Indian coal power plants are struggling under huge legacy costs as well as under cost of environmental compliance. If they are encouraged to integrate with solar under a public-private partnership structure they can find some breather. This will benefit old thermal power plants of small to medium capacity in many ways. First, they get acquainted with new technology, begin to get the knowledge of operation and economics and would also get to know how to work in a partnership. The private players get the security of their installation within the walled campus of the power plants. This can even lead them to joining hands in embracing new ventures on profit sharing basis.

But, the challenges do exist. Even at the cost of saving the site, there would be emissions from obtaining these materials and during manufacturing. Both solar and wind technologies produce intermittent power which must be addressed through redundancies, energy storage, or the use of natural gas or coal -fired turbines to supplement generation during low output (i.e., no sunshine or wind). Understanding the trade-offs involved in alternative electricity production technologies is important for ensuring that the future electricity supply is cost-effective, sustainable, and reliable. While energy production technologies differ with respect to these and other attributes, it is important to note that the electric grid is fed by a portfolio of technologies and that this portfolio is designed to take advantage of the strengths and weaknesses of each technology. 